\title{
Telomerase activity and telomere length in the colorectal polyp-carcinoma sequence
}

\author{
C. Valls Bautista ${ }^{1}$, C. Piñol Felis ${ }^{1}$, J. M. Reñe Espinet ${ }^{1,3}$, J. Buenestado García $^{1,3}$ and J. Viñas Salas ${ }^{2,4}$ \\ Departments of ${ }^{1}$ Medicine and ${ }^{2}$ Surgery. University of Lleida. Departments of ${ }^{3}$ Gastroenterology and ${ }^{4}$ Surgery. University \\ Hospital Arnau de Vilanova. IRB Lleida, Spain
}

\begin{abstract}
Objective: the role of telomerase activity and telomere length in the adenoma-carcinoma sequence of colon carcinogenesis has not been well established. The objective of this study was to determine telomerase activity and telomere length patterns in patients with adenomatous polyps either associated or not with colorectal cancer, as well as the role of telomeric instability in the adenomacarcinoma sequence.

Patients and methods: we included in the study 14 patients who underwent surgery for colorectal cancer and/or polyps. In 6 of these patients fresh samples of tumor tissue, polyps, and normal mucosa were obtained; in the 8 remaining cases, we collected only polyps and normal mucosa. We used the fluorescent-telomeric repeat amplification protocol assay (TRAP-F) to determine telomerase activity and telomere length using Southern-blot testing.

Results: telomerase activity was detected in $86 \%$ of polyps and $50 \%$ of associated normal mucosa. Mean telomerase activity in polyp tissue was 5.85 ; in the normal mucosa it was 0.58 TPG. Mean telomere length was $6.78 \mathrm{Kbp}$ and 7.78 , respectively. Polyps in patients without synchronous cancer had a telomerase activity that was significantly higher (9.4) than in those with cancer (1.1).

Conclusions: telomerase activity increases in the colorectal adenoma-carcinoma sequence, concurrently with a decrease in telomere length. The presence of synchronous cancer modifies telomerase activity in polyps.
\end{abstract}

Key words: Telomerase activity. Telomere length. Colorectal. Carcinoma. Polyp.

Supported by grants from Fondo de Investigaciones Sanitarias (01/0620) and FEDER. Cristina Valls is supported by a predoctoral grant by Generalitat de Catalunya.

Received: 05-01-09.
Accepted: $15-01-09$.

Correspondence: Carme Piñol Felis. Departamento de Medicina. Universitat de Lleida. Hospital Universitario Arnau de Vilanova. C/ Alcalde Rovira Roure, 80.25198 Lleida, Spain.e-mail: pinyol@medicina.udl.cat
Valls Bautista C, Piñol Felis C, Reñe Espinet JM, Buenestado García J, Viñas Salas. J. Telomerase activity and telomere length in the colorectal polyp-carcinoma sequence. Rev Esp Enferm Dig 2009; 101: 179-186.

\section{INTRODUCTION}

Currently, there is clinical, epidemiological and experimental evidence clearly indicating that polyps or adenomas are precursor lesions for most colorectal malignancies. However, this does not mean that all polyps evolve into cancer (1). From the colorectal cancer progression model proposed by Fearon and Volgestein (2) in 1990, several studies have established the changes apparent in the adenoma-carcinoma sequence. The main changes are mutations, deletions, and loss of heterozygosity (1,3-6).

Telomeres are structures located at the end of chromosomes in eukaryotic cells (7). They regulate gene expression, participate in the replication of chromosomes, and protect them from degradation by nucleases. In somatic cells, telomeres shorten progressively with each successive cell division. This progressive shortening is an important mechanism in the timing of human cellular aging. When telomeres become sufficiently short undergo a growth arrest called senescence phase, followed by apoptosis and death. Some cells escape from this mechanism and proliferate indefinitely by the action of telomerase. When these cells accumulate sufficient mutations affecting the cell cycle, they can become cancerous. Telomerase is a ribonucleoprotein that compensates the shortening of telomeres by reverse transcription using its intrinsic RNA as a template (8). In germ cells and tissues with high rates of renewal, telomerase is active and main- 
tains genome integrity and stability ensuring the transmission of total telomere length in each cell division (9). Telomerase activity was detected in $80-90 \%$ of human cancers, including colorectal cancer $(10,11)$, while its expression in the normal mucosa was $86 \%$ (12).

The role of telomeric dysfunction in the adenoma-carcinoma sequence has not been well established. The results available so far are divergent, and few studies are found in the literature comparing telomerase activity (TA) and telomere length (TL) in polyps and the normal mucosa of one same patient (13).

The first objective of this study was to determine the behavior of telomerase activity and telomere length in patients with polyps associated or otherwise with colorectal cancer, and the second objective was to explore the role of telomeric instability in the adenoma-carcinoma sequence, as defined by telomerase activity being higher in polyps than in adjacent normal mucosa, accompanied by changes or variations in telomere length.

\section{PATIENTS AND METHODS}

This preliminary study included 14 patients undergoing surgery for colorectal cancer and/or for endoscopically non-resectable polyps at Hospital Universitari Arnau de Vilanova, Lleida (Spain). Six of these patients had colonic tumors, polyps, and macroscopically normal mucosa $10 \mathrm{~cm}$ away from the tumor, while the remaining $8 \mathrm{sam}-$ ples had polyps and normal mucosa.

All samples were frozen at $-80{ }^{\circ} \mathrm{C}$ until their analysis. The research protocol was approved by the Hospital's Ethics Committee.

Telomerase activity was measured by a quantitative system using the fluorescent-telomeric repeat amplification protocol assay (F-TRAP) $(14,15)$, and the TRAPeze Telomerase Detection Kit (INTERGEN ${ }^{\circledast}$ Purchase, NY). The methodology was performed using the TRAP technique previously described (10).

Telomere length determination was performed by Southern blot using the TeloTAGGG Telomere Length Assay kit (Roche Diagnostics GMBH Mannheim). Briefly, 2 micrograms of genomic DNA were digested with Hinf I and Rsa I restriction enzymes, and the fragments separat- ed on 0.8 agarose gel. The DNA fragments were transferred onto a nylon membrane and hybridized to a specific probe for telomeric repeats (TTAGGG) digoxigenin- $_{4}$ conjugated. The hybridized probe was incubated with a digoxigenin specific antibody covalently coupled to alkaline phosphatase. Finally, the immobilized telomere probe was visualized using a highly sensitive chemiluminescent substrate for alkaline phosphatase. Chemiluminescence was detected by the system based on a DDC camera (Lumi-Imager), and analyzed with the Lumi-Analyst software (Boehringer Mannheim).

Telomere length was estimated according to $\Sigma(\mathrm{ODi}) /$ $\Sigma(\mathrm{ODi} / \mathrm{Li})$, where ODi is the intensity of the chemiluniscent signal and $\mathrm{Li}$ is the length of TRF fragments at point i. The unit of telomere length was Kilo-base pairs (Kbp). TRF lengths were recorded as telomere length.

The statistical analysis was performed using the program SPSS 14.0 (SPSS, Inc., Chicago, IL). Results of telomerase activity and telomere length are expressed as mean values. A mean comparison was calculated using Student's t-test, Mann-Whitney U test, and Kruskal-Wallis test. $\mathrm{p} \leq 0.05$ was considered statistically significant.

\section{RESULTS}

The present pilot study includes 14 patients with colorectal polyps, of which 6 showed synchronous colorectal polyps and cancer, while the remaining 8 were isolated polyps. All samples had telomerase activity and telomere length measured.

-Patients with colorectal polyps $(n=14)$. Telomerase activity expression was detected in $86 \%(12 / 14)$ of polyps and $50 \%(7 / 14)$ of their corresponding normal mucosa. Mean TA in polyps was 5.85 TPG and average normal mucosal 0.58 TPG, the difference did not reach significance $(\mathrm{p}=0.077)$.

We observed a significantly lower TA in polyps in patients with synchronous colorectal cancer (1.10 TPG) versus those who had no cancer (9.42 TPG), $\mathrm{p}=0.020$ (Table I).

Average telomere length in polyps was $6.78 \mathrm{Kbp}$, and $7.78 \mathrm{Kbp}$ in the normal mucosa; this difference did not reach significance $(\mathrm{p}=0.082)$ (Table $\mathrm{I})$.

Table I. Relationship of telomerase activity (TA) and telomere length in polyps and normal mucosa, both overall and in patients with or without synchronous cancer. Values expressed as mean and range

\begin{tabular}{|c|c|c|c|c|c|c|}
\hline & $\begin{array}{c}\text { Telomerase activity } \\
\text { polyp }\end{array}$ & $\begin{array}{c}\text { Telomerase activity } \\
\text { normal }\end{array}$ & Sig. $p$ & $\begin{array}{c}\text { Telomere lenght } \\
\text { polyp }\end{array}$ & $\begin{array}{c}\text { Telomere lenght } \\
\text { normal }\end{array}$ & Sig. $p$ \\
\hline$n=14$ & $5.85(0-33.40)$ & $0.58(0-2.35)$ & 0.077 & $6.78(3.21-6.78)$ & $7.78(4.83-7.78)$ & 0.082 \\
\hline \multicolumn{7}{|l|}{ Cancer } \\
\hline Yes (6/14) & $1.10(0-3.58)$ & $0.33(0-1.64)$ & 0.242 & $6.96(4.94-9.91)$ & $7.51(4.83-9.05)$ & 0.269 \\
\hline No $(8 / 14)$ & $9.42(0.91-33.4)$ & $0.77(0-2.35)$ & 0.097 & $6.65(3.21-11.19)$ & $7.98(5.64-11.33)$ & 0.173 \\
\hline$p$ & 0.020 & 0.345 & & 0.852 & 0.950 & \\
\hline
\end{tabular}


- Patients with synchronous colorectal cancer $(n=6)$. In all, $83 \%$ of tumors $(5 / 6), 67 \%$ of polyps $(4 / 6)$, and $33 \%$ of normal mucosa $(2 / 6)$ samples were telomerasepositive. Telomerase activity in tumors was $5.65 \mathrm{TPG}(0-$ 19.02), in polyps 1.09 (0-3.57), and in the normal mucosa 0.33 TPG (0-1.64). Differences between means were not significant but showed a higher TA in tumors than in polyps, and in polyps $v s$. normal mucosa (Fig. 1).

Average telomere length in tumors was $6.28 \mathrm{Kbp}$ (4.20-8.80), in polyps $6.95 \mathrm{Kbp}$ (4.94-9.91), and in the normal mucosa $7.50 \mathrm{Kbp}$ (4.83-9.05), without any significant differences (Fig. 1).

- Patients with isolated polyps $(n=8)$. Telomerase activity was detected in $100 \%(8 / 8)$ of polyps and $62.5 \%$ (5/8) of their corresponding normal mucosa. Mean telomerase activity in polyps was 9.41 TPG (0.91-33.41), and 0.77 in the normal mucosa TPG $(0-2.35)$, but the difference was not significant between them. Mean telomere length in polyps was $6.65 \mathrm{Kbp}$ (3.21-11.19), and 7.98 in the adjacent normal mucosa Kbp (5.64-11-33), without significant differences.

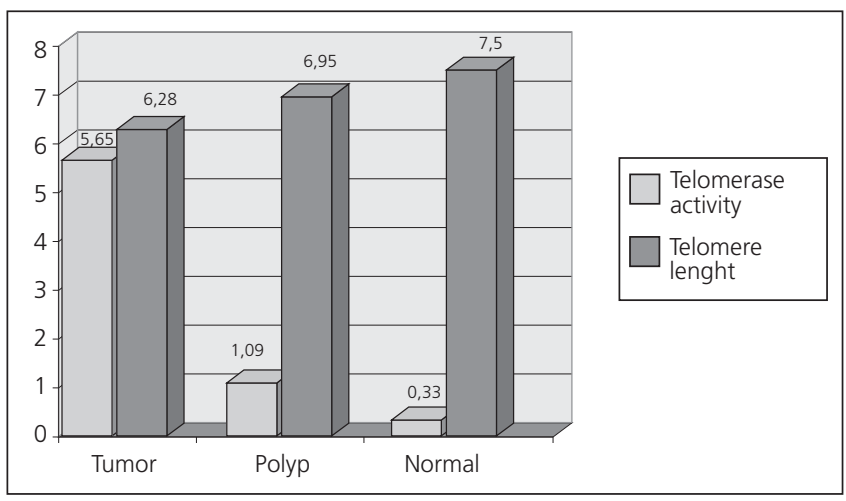

Fig. 1. Telomerase activity (unit: TPG) and telomere length (TRF) (unit: $\mathrm{Kbp}$ ) in the tumor, polyp and adjacent normal mucosa of patients with synchronous polyps and cancer.

Actividad de la telomerasa (unidad: TPG) y longitud del telómero (TRF) (unidad: Kbp) en el tumor, pólipo y mucosa adyacente normal de los pacientes con pólipos y cáncer sincrónico.

\section{DISCUSSION}

In our study $86 \%$ of polyps expressed telomerase activity, as well as $50 \%$ of cases in their adjacent normal mucosa. In one of the few published studies that compared polyps with normal mucosa in the same patients, the authors obtained TA expression in $22 \%$ of colorectal polyps, and none of all normal mucosa specimens (16). Other studies that compare TA in polyps with the normal mucosa of different patients found that polyp TA oscillates between 40 and $62.5 \%$, these values being 0 and $18.5 \%$ in the normal mucosa $(17-21)$. Related to those studies we observed a higher TA percentage in polyps and normal mucosa, which we believe likely due to the technique we used, fragment analysis by sequencing, which is very sensitive and can detect very low telomerase activities possibly common in the normal mucosa, and that can be missed by other methodologies.

Mean telomerase activity in polyps was 5.85 TPG, while it was 0.58 TPG in the adjacent normal mucosa. Other authors published polyp TA figures that oscillate between 1.7 and 7.8 TPG $(17,20)$ in the normal mucosa, from 0 to 3.34 TPG. None of these studies showed significant differences between polyp TA and normal mucosa TA (17-20).

Polyps synchronic with tumors have a significantly lower telomerase activity when compared to isolated polyps. Paradoxically, those results are accompanied by greater telomere length in the former. We think that this is due to the activation of alternative mechanisms for the maintenance of telomere length (ATL), which act with no increase in activity $(22,23)$.

Mean polyp telomere length was $6.78 \mathrm{Kbp}$ and 7.78 $\mathrm{Kbp}$ in the normal mucosa (difference did not reach significance). Mean values obtained in other studies in which the normal mucosa was compared with polyps of different patients, were as follows: Engelhardt et al. (20) $7.1 \mathrm{Kbp}$ in polyps and 7.46 Kbp in normal mucosa, Kim et al. (24) obtained 9,41 Kbp in polyps and 9.25 $\mathrm{Kbp}$ in normal mucosa, with no significant differences in both cases. Differences in mean telomere length found by those authors between polyps and normal mucosa were very small when compared to those in our study, possibly due to the fact that they did not work with paired samples.

We also studied the normal - adenoma - carcinoma sequence by measuring TA and TL in samples from 6 patients who had synchronous polyps and colorectal cancer, in which we also analyzed the normal mucosa. This part of the study could not be compared with other similar studies as we found none the literature.

TA was found in $83 \%(5 / 6)$ of tumors, in $67 \%(4 / 6)$ of polyps, and in $33 \%(2 / 6)$ of normal mucosa samples. Mean TA in tumor samples was 5.65 TGP, 1.09 TPG in polyps, and 0.53 TPG in normal mucosa. Those differences did not reach significance. We observed intermediate TA in polyps lower than in cancer but higher than in normal mucosa. We can conclude that there exists a progressive increase in TA levels in the sequence normal mucosa - adenoma - carcinoma, accompanied by a diminution of telomere length. We observed a tendency in the sequence normal tissue-adenoma-carcinoma where TA increases with cell malignancy grade, and telomere length diminishes. This behavior we think it due to the fact that the cell division ratio also increases with the passage from one tissue type to the next. We cannot compare those results because we found no similar studies in the literature. 
We think that with more cases those differences could be significant and confirm the existence of a sequence in telomere behavior (TA and TL) from normal towards tumoral mucosa through polyps. We believe that for a study of the sequence adenoma-carcinoma able to obtain coherent results it is necessary that mucosa samples showing the various stages of carcinogenic progression be studied in the same patients. With this approach we eliminate possible differences between individual patients and can compare the same number of cases for each type of mucosa. On the other hand, it allows to study the sequence in each patient individually.

In all 8 patients with isolated polyps we observed the same behavior as in all polyps taken together or in polyps synchronic with tumors: a higher TA in polyps than in the normal mucosa and lower telomere length in polyps versus the normal mucosa.

Although this is a preliminary study we can conclude that there is a growing telomerase activity in the adenoma-carcinoma sequence in the colonic mucosa, as well as a decrease in telomere length as we move toward the normal mucosa from the tumor. The presence of synchronous cancer modifies polyp telomerase activity.

In colorectal cancer there is an expression of telomerase that has been shown in previous studies. In the adenoma-cancer sequence we have demonstrated that there is obvious telomeric dysfunction. This finding would be a mechanism taking into account the changes already known to occur in this sequence, including deletions, mutations, oncogene activation, suppressor genes, or loss of heterozygosity.

\section{REFERENCES}

1. Ponz de Leon M, Di Gregorio C. Pathology of colorectal cancer. Dig Liver Dis 2001; 33(4): 372-88.

2. Fearon ER, Vogelstein B. A genetic model for colorectal tumorigenesis. Cell 1990; 61(5): 759-67.

3. Cruz-Bustillo Clarens D. Génetica molecular del cáncer colorectal. Rev Esp Enferm Dig 2004; 96(1): 48-59.

4. Cheng L, Lai MD. Aberrant crypt foci as microscopic precursors of colorectal cancer. World J Gastroenterol 2003; 9(12): 2642-9.

5. Benito M, Díaz-Rubio E. Molecular biology in colorectal cancer. Clin Trans Oncol 2006; 8(6): 391-8.

6. Fenoglio-Preiser CM, Noffsinger A. Aberrant crypt foci: A review.
Toxicol Pathol 1999; 27(6): 632-42.

7. Blackburn EH. Structure and function of telomeres. Nature 1991; 350(6319): 569-73.

8. Shay JW, Zou Y, Hiyama E, Wright WE. Telomerase and cancer. Hum Mol Genet 2001; 310(7): 677-85.

9. Hiyama E, Hiyama K. Telomerase as tumor marker. Cancer Lett 2003; 194(2): 221-33.

10. Bautista CV, Felis CP, Espinet JM, Salas J. Telomerase activity is a prognostic factor for recurrence and survival in rectal cancer. Dis Colon Rectum 2007; 50(5): 611-20

11. Saleh S, Lam AK, Ho YH. Real-time PCR quantification of human telomerase reverse transcriptase (hTERT) in colorectal cancer. Pathology 2008; 40: 25-30.

12. Kammori M, Kanauchi H, Nakamura K, Kawahara M, Weber TK, Mafune KI, et al. Demonstration of human telomerase reverse transcriptase in human colorectal carcinoma by in situ hybridization. Int J Oncol 2002; 20(1): 15-21.

13. Boldrini L, Faviana P, Gisfredi S, Zucconi Y, Di Quirico D, Donati $\mathrm{V}$, et al. Evaluation of telomerase in the development and progression of colon cancer. Int J Mol Med 2002; 10(5): 589-92.

14. Kim NW, Piatyszek MA, Prowse KR, Harley CB, West MD, Ho PL, et al. Specific association of human telomerase activity with immortal cells and cancer. Science 1994; 266(5193): 2011-5.

15. Piatyszek MA, Kim NW, Weinrich SL, Hiyama K, Wright WE, Shay JW. Detection of telomerase activity in human cells and tumors by a telomeric repeat amplification protocol (TRAP). Methods Cell Sci 1995; 17: 1-15

16. Katayama S, Shiota G, Oshimura M, Kawasaki H. Clinical usefulness of telomerase activity and telomere length in the preoperative diagnosis of gastric and colorectal cancer. J Cancer Res Clin Oncol 1999; 125(7): 405-10

17. Abe N, Watanabe T, Nakashima M, Masaki T, Mori T, Sugiyama M, et al. Quantitative analysis of telomerase activity: a potential diagnostic tool for colorectal cancer. Hepatogastroenterology 2001; 48(39): 692-5

18. Yan P, Saraga EP, Bouzourene H, Bosman FT, Benhattar J. Telomerase activation in colorectal carcinogenesis. J Pathol 1999; 189(2): 207-12.

19. Tang R, Cheng AJ, Wang JY, Wang TC. Close correlation between telomerase expression and adematous polyp progression in multistep colorectal carcinogenesis. Cancer Res 1998; 58(18): 4052-4.

20. Engelhardt M, Drullinsky P, Guillem J. Telomerase and telomere length in the development and progression of premalignant lesions to colorectal cancer. Clin Cancer Res 1997; 122(11): 1141-5.

21. Mizumoto I, Ogawa Y, Niiyama H, Nagai E, Sato I, Urashima T, et al. Possible role of telomerase activation in the multistep tumor progression of periampullary lesions in patients with familial adenomatous polyposis. Am J Gastroenterol 2001; 96(4): 1261-5.

22. Bechter OE, Zou Y, Walter W, Wright WE, Shay JW. Telomeric recombination in mismatch repair deficient human colon cancer cells after telomerase inhibition. Cancer Res 2004; 64(10): 344451 .

23. Nittis T, Guittat L, Stewart SA. Alternative lengthening of telomeres (ALT) and chromatin: is there a connection? Biochimie 2008; 90(1): $5-12$.

24. Kim HR, Kim YJ, Kim HJ, Kim SK, Lee JH. Telomere length changes in colorectal cancers and polyps. J Korean Med Sci 2002; 17(3): $360-5$ 